\title{
VALIDATION OF A WEB APP FOR AUTOMATION OF ROBSON DATA COLLECTION FOR OBSTETRIC AUDITS IN A PROFESSORIAL OBSTETRIC UNIT OF A TERTIARY CARE HOSPITAL IN SRI LANKA: OBSERVATIONAL STUDY
}

\author{
Chandana Jayasundara ${ }^{1}$, Indu Jayawardane ${ }^{2}$, and Ananda Perera ${ }^{3}$ \\ ${ }^{1}$ University of Colombo Faculty of Medicine \\ ${ }^{2}$ University of Colombo, Sri Lanka. \\ ${ }^{3}$ Biosoft
}

April 28, 2020

\begin{abstract}
ABSTRACT (223) Objective Validation of a webApp developed to automate the Robson classification and the WHO audit on rising Caesarean section rates Materials and Methods RobsApp will classify all the mothers admitted prospectively. The program generates an audit report based on the WHO criteria. Results Frequency distribution of the Caesarean section rates among the Robson groups was similar to the multicountry survey report. The Pearson's correlation coefficient between the RobsApp and Robsons's study was 0.752 4, Senanyake et al.'s study 0.8705, Keisuke and Kassam et al.'s study 0.7314. All correlations were significant at $<0.05$. Discussion The results prove the validity of the RobsApp. The results are also broadly in agreement with the trends found in other studies reported in the literature. Therefore RobsApp may be used to analyze the Caesarean section rates of a given obstetric unit. The ease of use and convenient integration into routine ward workflow also suggest the outcome studies based on continuous audits are feasible. The App can also be used to analyze the intra-unit trends in Caesarean section rates as well as inter-unit, regional or even the national Caesarean section rates. Conclusion The RobsApp can confidently be used in obstetric units anywhere for the audit and outcome follow ups of caesarean section rates.
\end{abstract}

\section{Hosted file}

bjog-ValidationWebApp.docx available at https://authorea.com/users/314850/articles/445240-validationof-a-web-app-for-automation-of-robson-data-collection-for-obstetric-audits-in-a-professorialobstetric-unit-of-a-tertiary-care-hospital-in-sri-lanka-observational-study 


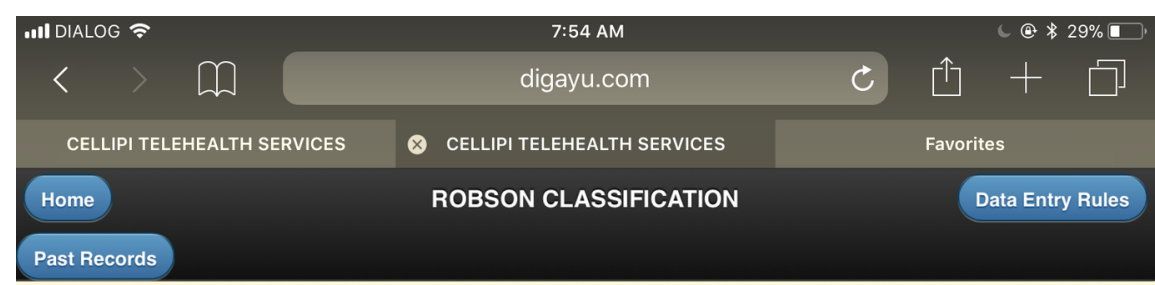

\begin{tabular}{|c|c|}
\hline Username & \\
\hline Password & \\
\hline First Name/Clinicld & \\
\hline Last Name & \\
\hline Age & \\
\hline PARITY : & - NO INFO \\
\hline & Nullipara \\
\hline & Multipara \\
\hline PREVIOUS CS & - NO INFO \\
\hline & No past CS \\
\hline & Past CS ONCE \\
\hline & Past CS MORE THAN ONCE \\
\hline $\begin{array}{l}\text { ONSET OF } \\
\text { LABOUR : }\end{array}$ & - NO INFO \\
\hline & Spontaneous \\
\hline & Induced \\
\hline & No labour \\
\hline NO OF FETUSES : & - Singleton \\
\hline & Multiple \\
\hline $\begin{array}{l}\text { GESTATIONAL AGE } \\
:\end{array}$ & - NO INFO \\
\hline & More than 37 \\
\hline
\end{tabular}




\begin{tabular}{|c|c|c|}
\hline .II DIALOG ₹ & $\begin{array}{l}\text { 7:54 AM } \\
\text { digayu.com }\end{array}$ & 아 $* 29 \%$ 口 \\
\hline \multirow{4}{*}{$\begin{array}{l}\text { DELIVERY } \\
\text { OUTCOME : }\end{array}$} & ( Live birth & \\
\hline & Still birth & \\
\hline & T2 miscarriage & \\
\hline & Multiple birth & \\
\hline \multirow{5}{*}{$\begin{array}{l}\text { DELIVERY } \\
\text { COMPLICATIONS : }\end{array}$} & ( FTND & \\
\hline & (1) $\mathbf{P P H}$ & \\
\hline & (1) Retained Placenta & \\
\hline & (1) Prolonged Labor & \\
\hline & (1) Neonatal Problems & \\
\hline \multirow{8}{*}{$\begin{array}{l}\text { CS ELECTIVE } \\
\text { INDICATIONS : }\end{array}$} & ( no elective CS & \\
\hline & past $\mathrm{CS}$ and failed VBAC & \\
\hline & breech and failed ECV & \\
\hline & breech and primi & \\
\hline & Multiple Fetuses and T2 breech & \\
\hline & large baby & \\
\hline & transverse or oblique lie & \\
\hline & (1) maternal request & \\
\hline \multirow{7}{*}{$\begin{array}{l}\text { CS EMERGENCY } \\
\text { INDICATIONS : }\end{array}$} & ( no emergency CS & \\
\hline & CTG abnormalities & \\
\hline & meconium stained liquor & \\
\hline & thick meconium & \\
\hline & failure to progress in spontaneous labor & \\
\hline & failure to progress in induced labor & \\
\hline & PET or medical complication of pregnancy & \\
\hline
\end{tabular}




\begin{tabular}{|c|c|}
\hline all DIALOG & $\begin{array}{l}\text { 7:54 AM } \\
\text { digayu.com }\end{array}$ \\
\hline \multirow[t]{8}{*}{$\begin{array}{l}\text { CS ELECTIVE } \\
\text { INDICATIONS : }\end{array}$} & - no elective CS \\
\hline & past CS and failed VBAC \\
\hline & breech and failed ECV \\
\hline & breech and primi \\
\hline & Multiple Fetuses and T2 breech \\
\hline & large baby \\
\hline & transverse or oblique lie \\
\hline & maternal request \\
\hline \multirow{8}{*}{$\begin{array}{l}\text { CS EMERGENCY } \\
\text { INDICATIONS : }\end{array}$} & - no emergency cS \\
\hline & CTG abnormalities \\
\hline & meconium stained liquor \\
\hline & thick meconium \\
\hline & failure to progress in spontaneous labor \\
\hline & failure to progress in induced labor \\
\hline & PET or medical complication of pregnancy \\
\hline & fetal growth retardation severe \\
\hline Notes & \\
\hline & SAVE \\
\hline & DATA ANALYSIS \\
\hline
\end{tabular}




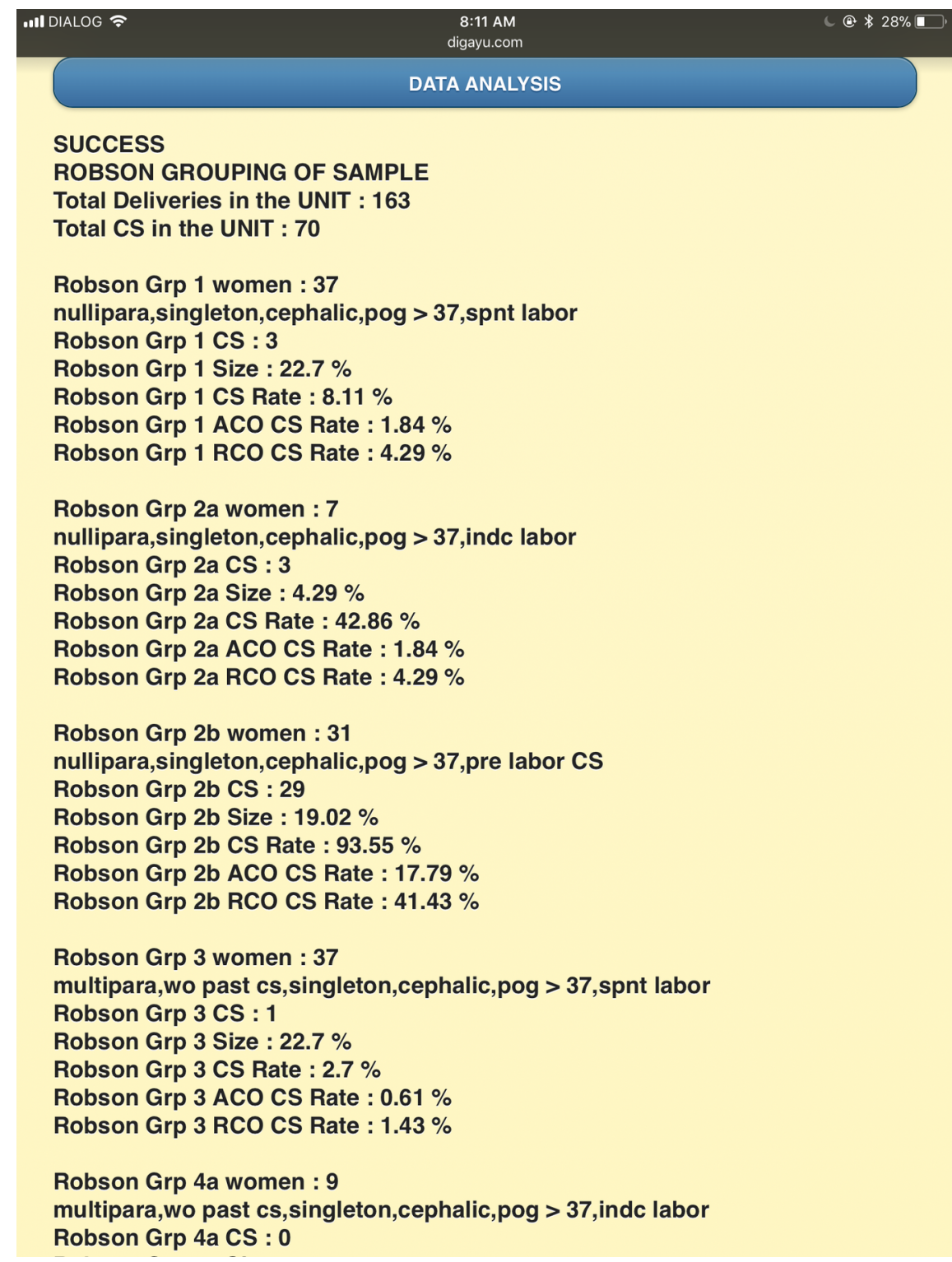

